\title{
A VARIATION PRINCIPLE FOR GROUND SPACES
}

\author{
STEPHAN WEIS
}

\begin{abstract}
The ground spaces of a vector space of hermitian matrices, partially ordered by inclusion, form a lattice constructible from top to bottom in terms of intersections of maximal ground spaces. In this paper we characterize the lattice elements and the maximal lattice elements within the set of all subspaces using constraints on operator cones. Our results contribute to the geometry of quantum marginals, as their lattices of exposed faces are isomorphic to the lattices of ground spaces of local Hamiltonians.
\end{abstract}

\section{INTRODUCTION}

The variation principle [27, 51] for estimating the ground energy $E_{0}$ (smallest eigenvalue) of a Hamiltonian $h$ (hermitian matrix) asserts that

$$
E_{0} \leqslant\langle\psi|h| \psi\rangle
$$

for all pure states $|\psi\rangle$. This means that $E_{0}$ is the smallest expected energy a quantum system can have in any pure state $|\psi\rangle$ if $h$ is the energy operator. The upper bound on $E_{0}$ is mathematically trivial but computationally very useful. Nevertheless, the estimation of $E_{0}$ for the class of $k$-local Hamiltonians, describing the energy of a many-body system without interactions between more than $k$ units, is a hard problem already for $k=2$ [36, 35, 20]. Geometric approaches to the local Hamiltonian problem of estimating $E_{0}$ are discussed since the 1960's. A basic idea is that $E_{0}$ is the displacement of the supporting hyperplane with normal vector $h$ touching the convex set $\mathcal{D}_{(k)}$ of $k$-body marginals.

Similar to the local Hamiltonian problem, the quantum marginal problem of deciding whether a tuple of states lies in $\mathcal{D}_{(k)}$ is neither believed to have efficiently computable solutions [39, 60]. Many of the results pertaining to $\mathcal{D}_{(k)}$ were originally obtained in the fermionic case (not treated here). Notably, spectral properties of marginal density matrices [19, 3, 52, 18] were discovered. Spectral conditions are insufficient to characterize $k$-body marginals of overlapping subsystems $(k>1)$, for that there are results concerning extreme points [19, 23, 45, 17] and order-theoretic results regarding ground spaces of marginal states [16].

In this article we study order-theoretic aspects of the convex geometry of a set of quantum marginals - we begin with linear images of general convex sets, then linear images of state spaces of matrix algebras, and finally the very special case of quantum marginals $\mathcal{D}_{(k)}$. More precisely, a quantum mechanical system [15, 2] is described by a complex *-subalgebra of $M_{n}$ including the $n$-by- $n$ identity matrix $\mathbb{1}$. A density matrix, or state, of $\mathcal{A}$ is a matrix of trace one in the cone $\mathcal{A}^{+}:=\{a \in \mathcal{A}: a \geq 0\}$ of positive semi-definite matrices in $\mathcal{A}$. The states of $\mathcal{A}$ form a convex set $C_{\mathcal{A}}$, called state space.

2010 Mathematics Subject Classification. Primary 52A20, 52B05, 51D25, 47L07, 47A12, 81P16. Secondary 62F30, 94A17.

Key words and phrases. convex set, exposed face, normal cone, variation principle, quantum marginals, local Hamiltonian, ground space, operator cone, coatom. 
According to the variation principle (1.1) and using a simple convexity argument, the ground energy of a Hamiltonian $u \in \mathcal{A}$ is

$$
\min \left\{\langle\rho, u\rangle: \rho \in C_{\mathcal{A}}\right\},
$$

where $\langle a, b\rangle=\operatorname{tr}\left(a^{*} b\right)$ is the Hilbert-Schmidt inner product of $a, b \in \mathcal{A}$. Assuming $u$ lies in a linear subspace $U \subset \mathcal{A}$ of hermitian matrices, Euclidean geometry shows

$$
\min \left\{\langle b, u\rangle: b \in \pi\left(C_{\mathcal{A}}\right)\right\}, \quad u \in U,
$$

where $\pi$ is the orthogonal projection onto $U$. Geometrically, equation (1.2) means that the ground energy restricted to $U$ is the distance of the origin from the supporting hyperplane of $\pi\left(C_{\mathcal{A}}\right)$ with inner normal vector $u$.

A well-known aspect of $\pi\left(C_{\mathcal{A}}\right)$ is the isomorphism between its exposed faces and the ground projections of $U$. An exposed face of $\pi\left(C_{\mathcal{A}}\right)$ is the intersection of $\pi\left(C_{\mathcal{A}}\right)$ with a supporting hyperplane, that is the subset of $\pi\left(C_{\mathcal{A}}\right)$ at which the minimum (1.2) is achieved for some $u \in U$. The ground projection $p_{0}(u)$ of $u$ is the spectral projection of $u$ corresponding to the smallest eigenvalue. The ground space of $u$ is the image of $p_{0}(u)$. The set of exposed faces of $\pi\left(C_{\mathcal{A}}\right)$, partially ordered by inclusion, is lattice-isomorphic to the set $\mathcal{P}(U)$ of ground projections of $U$, partially ordered by the Löwner partial ordering [57].

A discussion of normal cones of $\pi\left(C_{\mathcal{A}}\right)$ leads to the Definition 5.2 of the cone

$$
K(p):=p^{\prime} \mathcal{A}^{+} p^{\prime} \cap U
$$

for projections $p \in \mathcal{A}$, that is $p=p^{*}=p^{2}$. Thereby, $p^{\prime}=\mathbb{1}-p$ is the complementary projection of $p$, and $p^{\prime} \mathcal{A}^{+} p^{\prime}=\left\{p^{\prime} a p^{\prime}: a \in \mathcal{A}^{+}\right\}$. Notice that

$$
K(p)=\{u \in U \mid u \geq 0, p \leq \operatorname{ker}(u)\},
$$

where $\operatorname{ker}(u)$ is the kernel projection of $u$. It is easy to show that if $\mathbb{1} \in U$, then the ground projection $p_{0}(u)$ of any $u \in U$ is greater than or equal to any projection $p \in \mathcal{A}$ satisfying $K(p) \supset K\left(p_{0}(u)\right)$. Theorem 5.4 completes this statement to what we call variation principle: For every projection $p \in \mathcal{A}$, the set of projections $q \in \mathcal{A}$ satisfying $K(q)=K(p)$ has a greatest element $q_{\max }(p)$ and $q_{\max }(p) \in \mathcal{P}(U)$.

The variation principle shows that the decision problem of whether a projection $p \in \mathcal{A}$ lies in $\mathcal{P}(U)$ is equivalent to that of whether $p=q_{\max }(p)$. One way to compute $q_{\max }(p)$ is to pick a relative interior point $u$ of $K(p)$ and compute its ground projection $p_{0}(u)$, as $q_{\max }(p)=p_{0}(u)$ follows from results concerning normal cones of state spaces [54]. This raises the question, not teated here, of whether a relative interior point of $K(p)$ can be efficiently computed.

The computation of $\mathcal{P}(U)$ can be put down to that of the maximal elements of $\mathcal{P}(U) \backslash\{\mathbb{1}\}$, known as coatoms, as the latter generate $\mathcal{P}(U)$ in terms of infima [57]. Clearly, a projection $p$ of $\mathcal{A}$ of rank $n-1$ is a coatom if and only if $p^{\prime} \in U$, that is if $K(p)$ is a ray. Theorem 6.1(1) proves for any $p \in \mathcal{P}(U)$ that $p$ is a coatom if and only if $K(p)$ is a ray. The interesting part of the theorem, that $p \in \mathcal{P}(U)$ is not a coatom if $\operatorname{dim}(K(p))>1$, is exploited in Section 8. Infima of projections are studied, for example, in [37, 26, 14].

The article is organized as follows. Section 2 proves a variation principle for convex sets. Sections 3 and 4 recall lattice isomorphisms related to the state space and its linear images. Section 5 translates the variation principle to ground spaces. Section 6 characterizes coatoms. Section 7 discusses a non-commutative example. Sections 8 and 9 deal with local Hamiltonians. Section 10 is a conclusion. 
Remark 1.1 (Complexity of $\mathcal{D}_{(k)}$ ). The semi-definite extension complexity [24, 5] of $\mathcal{D}_{(k)}$ is the minimal dimension $d$ for which $\mathcal{D}_{(k)}$ is a linear image of an affine section of $M_{d}^{+}$. The local Hamiltonian problem being hard for $k \geqslant 2$, the existence of efficient algorithms [43] for linear optimization on $M_{d}^{+}$suggests that the complexity $O\left(2^{N}\right)$ of $\mathcal{D}_{(k)}$ for $N$ qubits is unlikely to be polynomial in $N$. Because of the gap from $O\left(2^{N}\right)$ to the polynomial $\operatorname{dim}\left(\mathcal{D}_{(k)}\right)=\Theta\left(N^{k}\right)$, the set $\mathcal{D}_{(k)}$ has a much richer convex geometry than sections and projections of $M_{d}^{+}$can have for $d$ of order $\sqrt{N^{k}}$.

Remark 1.2 (Topology of $\mathcal{P}(U)$ ). In addition to contributing to the geometry of $\pi\left(C_{\mathcal{A}}\right)$, we hope that the results will ultimately enable us to discuss the topology of $\mathcal{P}(U)$. If the algebra $\mathcal{A}$ is non-commutative, then $\mathcal{P}(U)$ may not be closed in the norm topology, because of discontinuities of the maximum-entropy inference map $\pi\left(C_{\mathcal{A}}\right) \rightarrow C_{\mathcal{A}}$ under linear constraints [58]. For example, $\mathcal{P}\left(U_{(2)}\right)$ is not norm closed for three qubits [50]. For a many-body system, the discontinuity of the inference map $\mathcal{D}_{(k)} \rightarrow C_{\mathcal{A}}$ is equivalent [56, 59, 50] to that of a correlation quantity [4, 7, 61, 6, 48, 44, 30] closely related to the "irreducible correlation" first introduced in [38]. For $k=1$, the quantity is the mutual information or multi-information, which is continuous and which measures the total correlation [28].

\section{A VARIATION PRINCIPLE FOR PRE-IMAGES OF EXPOSED FACES}

We show that the pre-images of exposed faces of a projection of a convex set $C$ are the greatest exposed faces under a normal cone constraint.

Definition 2.1. A closure operation [1, 13] on a set $I$ is an operator $2^{I} \rightarrow 2^{I}$, $X \mapsto \operatorname{cl}(X)$ on the subsets of $I$ such that for all $X, Y \subset I$ we have $X \subset \operatorname{cl}(X)$ (extensive), $\operatorname{cl}(\operatorname{cl} X)=\operatorname{cl}(X)$ (idempotent), and $X \subset Y \Longrightarrow \operatorname{cl}(X) \subset \operatorname{cl}(Y)$ (isotone). Subsets $X \subset I$ with $X=\operatorname{cl}(X)$ are called closed sets with respect to cl.

Lemma 2.2. Let cl be a closure operation on a set $I$. Let $\mathcal{S} \subset 2^{I}$ contain all closed subsets of $I$. A set $X \in \mathcal{S}$ is closed with respect to cl if and only if $X$ is the greatest element of $\{G \in \mathcal{S} \mid \operatorname{cl}(G)=\operatorname{cl}(X)\}$, partially ordered by inclusion.

Proof: Let $X \in \mathcal{S}$ be closed and let $G \in \mathcal{S}$ such that $\operatorname{cl}(G)=\operatorname{cl}(X)$, then

$$
G \subset \operatorname{cl}(G)=\operatorname{cl}(X)=X .
$$

Conversely, let $X$ be the greatest element of all $G \in \mathcal{S}$ for which $\operatorname{cl}(G)=\operatorname{cl}(X)$ holds. Since $\operatorname{cl}(X) \in \mathcal{S}$ and since $\operatorname{cl}(\operatorname{cl} X)=\operatorname{cl}(X)$ we have $X \supset \operatorname{cl}(X)$. As cl is extensive, we obtain $X=\operatorname{cl}(X)$ which shows that $X$ is closed.

As a standard notation for the article, let $A$ denote a finite-dimensional Euclidean vector space, $C \subset A$ a convex subset, $U \subset A$ a linear subspace, and

$$
\pi_{U}: A \rightarrow A
$$

the orthogonal projection onto $U$. We frequently write $\pi$ instead of $\pi_{U}$. An exposed face of $C$ is defined to be either the empty set or any subset of $C$ of the form

$$
F_{C}(a):=\operatorname{argmin}\{\langle x, a\rangle \mid x \in C\}, \quad a \in A .
$$

We denote by $\mathcal{E}(C)$ the set of exposed faces of $C$. If $x \in C$ and $\{x\}$ is an exposed face, then $x$ is called an exposed point. 
Definition 2.3 (Complete lattice). Let $\varphi: \mathcal{K}_{1} \rightarrow \mathcal{K}_{2}$ be a map between two partially ordered sets $\mathcal{K}_{1}, \mathcal{K}_{2}$. The map $\varphi$ is isotone if $x \leqslant y \Longrightarrow \varphi(x) \leqslant \varphi(y)$ holds and $\varphi$ is antitone if $x \leqslant y \Longrightarrow \varphi(x) \geqslant \varphi(y)$ holds. Let $\mathcal{K}$ be a lattice, that is a partially ordered set where the infimum $k \wedge \ell$ and supremum $k \vee \ell$ of each pair of elements $k, \ell \in \mathcal{K}$ exists. The lattice $\mathcal{K}$ is complete if the infimum and supremum of an arbitrary subset of $\mathcal{K}$ exist. For a complete lattice $\mathcal{K}$, the infimum of $\varnothing$ is defined to be the greatest element of $\mathcal{K}$ and the supremum of $\varnothing$ is the smallest element.

The set of exposed faces $\mathcal{E}(C)$, partially ordered by inclusion, forms a complete lattice whose infimum is the intersection [8, 40, 55]. Consider the set of pre-images of exposed faces of $\pi(C)$,

$$
\mathcal{L}=\mathcal{L}_{C, U}=\left\{\left.\pi\right|_{C} ^{-1}(F) \mid F \in \mathcal{E}(\pi(C))\right\} .
$$

We denote the smallest element of $\mathcal{L}$ containing $X \subset C$ by

$$
\operatorname{cl}_{\mathcal{L}}(X)=\bigcap\{F \in \mathcal{L} \mid X \subset F\} .
$$

The set $\mathcal{L}$, partially ordered by inclusion, is again a complete lattice whose infimum is the intersection, for details see Proposition 5.6 of [55]. Hence, the set-valued map $\mathrm{cl}_{\mathcal{L}}: 2^{C} \rightarrow 2^{C}$ is a closure operation whose closed sets are the elements of $\mathcal{L}$. Clearly $\mathcal{L} \subset \mathcal{E}(C)$ holds so Lemma 2.2 shows the following.

Lemma 2.4. Let $F \in \mathcal{E}(C)$. We have $F \in \mathcal{L}$ if and only if $F$ is the greatest element of $\left\{G \in \mathcal{E}(C) \mid \operatorname{cl}_{\mathcal{L}}(G)=\operatorname{cl}_{\mathcal{L}}(F)\right\}$, partially ordered by inclusion.

Let us reformulate the condition $\operatorname{cl}_{\mathcal{L}}(G)=\operatorname{cl}_{\mathcal{L}}(F)$ of Lemma 2.4 in terms of normal cones. First, we recall from Proposition 5.6 of [55] that the projection

$$
\left.\pi\right|_{\mathcal{L}}: \mathcal{L} \rightarrow \mathcal{E}(\pi(C))
$$

is an isotone lattice isomorphism. Here, the set-valued map $2^{A} \rightarrow 2^{A}$ induced by the projection $\pi: A \rightarrow A$ is denoted by the symbol $\pi$, too. We denote the smallest exposed face of $C$ containing $X \subset C$ by

$$
\operatorname{cl}_{\mathcal{E}(C)}(X)=\bigcap\{F \in \mathcal{E}(C) \mid X \subset F\} .
$$

Lemma 2.5. For all $X \subset C$ we have $\pi\left(\operatorname{cl}_{\mathcal{L}}(X)\right)=\operatorname{cl}_{\mathcal{E}(\pi(C))}(\pi(X))$.

Proof: Using the lattice isomorphism of (2.4), one obtains from 2.3) the equation

$$
\pi\left(\operatorname{cl}_{\mathcal{L}}(X)\right)=\bigcap\left\{F \in \mathcal{E}(\pi(C))|X \subset \pi|_{C}^{-1}(F)\right\} .
$$

For $F \in \mathcal{E}(\pi(C))$ one has $\left.\pi \circ \pi\right|_{C} ^{-1}(F)=F$. Hence $\left.X \subset \pi\right|_{C} ^{-1}(F)$ implies $\pi(X) \subset F$. Conversely, $\pi(X) \subset F$ implies

$$
\left.\left.X \subset \pi\right|_{C} ^{-1} \circ \pi(X) \subset \pi\right|_{C} ^{-1}(F)
$$

which completes the proof.

A vector $u \in A$ is a normal vector (inward pointing) of $C$ at $x \in C$ if $\langle y-x, u\rangle \geqslant 0$ for all $y \in C$. The normal cone $N_{C}(x)$ of $C$ at $x$ is the set of all normal vectors of $C$ at $x$. The relative interior $\operatorname{ri}(C)$ of $C$ is the interior of $C$ in the topology of the affine hull of $C$. The normal cone $N_{C}(X)$ of $C$ at a non-empty convex subset $X \subset C$ is defined to be the normal cone of $N_{C}(x)$ of $C$ at any point $x \in \operatorname{ri}(X)$ (the definition does not depend on $x$, see [53, 55]). The normal cone of $C$ at $\varnothing$ is defined to be $N_{C}(\varnothing)=A$. Let

$$
\mathcal{N}(C):=\left\{N_{C}(X) \mid X \subset C \text { is convex }\right\}
$$

denote the set of normal cones of $C$. 
We recall basic properties of normal cones employed later. First, $\mathcal{N}(C)$ is, partially ordered by inclusion, a complete lattice whose infimum is the intersection [55]. Second, normal cones do not decrease under the closure operation $\mathrm{cl}_{\mathcal{E}(C)}$,

$$
N_{C}(X)=N_{C}\left(\operatorname{cl}_{\mathcal{E}(C)}(X)\right), \quad \text { for convex } X \subset C .
$$

The equation (2.5) is proved in Lemma 4.6 of [55] for faces $X$ of $C$, but is true as stated here $\mathrm{g}^{1}$ for convex subsets $X \subset C$. Thereby, a face of $C$ is a convex subset of $C$ which contains every closed segment contained in $C$ whose open segment it meets. Third, if $C$ is not a singleton, then Proposition 4.7 of [55] shows that

$$
N_{C}: \mathcal{E}(C) \rightarrow \mathcal{N}(C), \quad F \mapsto N_{C}(F)
$$

is an antitone lattice isomorphism. Fourth, we interpret normal cones of $\pi(C)$ as subsets of $U$. For $x \in \pi(C)$ and $\left.y \in \pi\right|_{C} ^{-1}(\{x\})$, the normal cone of $\pi(C)$ at $x$ is $N_{\pi(C)}(x)=N_{C}(y) \cap U$, see Lemma 5.9 of [55]. Hence

$$
N_{\pi(C)}(\pi(X))=N_{C}(X) \cap U, \quad \text { for convex } X \subset C,
$$

because $\pi($ ri $X) \subset \operatorname{ri}(\pi(X))$, see for example [49]. By convention, $N_{\pi(C)}(\varnothing)=U$.

Proposition 2.6. Let $\pi(C)$ be not a singleton and let $F \in \mathcal{E}(C)$. Then $F \in \mathcal{L}$ holds if and only if $F$ is the greatest element of $\left\{G \in \mathcal{E}(C) \mid N_{C}(G) \cap U=N_{C}(F) \cap U\right\}$, partially ordered by inclusion.

Proof: Let $F \in \mathcal{E}(C)$. Lemma 2.4 shows that $F$ lies in $\mathcal{L}$ if and only if $F$ is the greatest among all exposed faces $G \in \mathcal{E}(C)$ which have the same closure in $\mathcal{L}$,

$$
\operatorname{cl}_{\mathcal{L}}(G)=\operatorname{cl}_{\mathcal{L}}(F) \text {. }
$$

We transform the condition into five equivalent conditions.

$$
\begin{aligned}
\pi\left(\operatorname{cl}_{\mathcal{L}} G\right) & =\pi\left(\operatorname{cl}_{\mathcal{L}} F\right) \\
\operatorname{cl}_{\mathcal{E}(\pi(C))} \pi(G) & =\operatorname{cl}_{\mathcal{E}(\pi(C))} \pi(F) \\
N_{\pi(C)}\left(\operatorname{cl}_{\mathcal{E}(\pi(C))} \pi(G)\right) & =N_{\pi(C)}\left(\operatorname{cl}_{\mathcal{E}(\pi(C))} \pi(F)\right) \\
N_{\pi(C)}(\pi(G)) & =N_{\pi(C)}(\pi(F)) \\
N_{C}(G) \cap U & =N_{C}(F) \cap U .
\end{aligned}
$$

These conditions follow, respectively, from the isomorphisms 2.4 , Lemma 2.5, the isomorphism (2.6), equation (2.5), and equation (2.7).

\section{Geometric Representations of projections}

Classical statistics and the statistics of quantum mechanics are special among more general statistical theories [31, 29] because they can be described in the setting of $\mathrm{C}^{*}$-algebras. We recall some lattice isomorphisms in the $\mathrm{C}^{*}$-algebraic context [2].

Here and subsequently, let $\mathcal{A} \subset M_{n}$ be a complex *-subalgebra including the $n$-by$n$ identity matrix $\mathbb{1}$, and let $A \subset \mathcal{A}$ denote the real subspace of hermitian matrices. The algebra $\mathcal{A}$ is partially ordered by the relation $a \leq b, a, b \in \mathcal{A}$, also denoted $b \geq a$, which means that $b-a$ is positive semi-definite. This partial ordering is known as the Löwner partial ordering. The fundamental events of the statistical theory form the set of projections of $\mathcal{A}$,

$$
\mathcal{P}_{\mathcal{A}}:=\left\{p \in \mathcal{A} \mid p=p^{*}=p^{2}\right\} .
$$

\footnotetext{
${ }^{1}$ Lemma 4.4 of [55] and the last statement of Lemma 4.6 of [55] are formulated for faces, but the proofs are straight forward to generalize from faces to arbitrary convex subsets.
} 
The set $\mathcal{P}_{\mathcal{A}}$, endowed with the Löwner partial ordering, is a complete lattice [2] and

$$
\mathcal{P}_{\mathcal{A}} \rightarrow\left\{\operatorname{image}(p) \mid p \in \mathcal{P}_{\mathcal{A}}\right\}, \quad p \mapsto \operatorname{image}(p) \subset \mathbb{C}^{n}
$$

is a lattice isomorphism, where subspaces of $\mathbb{C}^{n}$ are partially ordered by inclusion. The infimum of two subspaces is their intersection.

In quantum mechanics, a hermitian matrix has the interpretation of energy operator (Hamiltonian) and its eigenvalues are the possible energy values [11. We denote the real vector space of hermitian matrices by

$$
A:=\left\{a \in \mathcal{A} \mid a^{*}=a\right\} .
$$

The smallest eigenvalue $\lambda_{0}(a)$ of $a \in A$ is the ground energy of $a$, the corresponding eigenspace of $a$ is the ground space of $a$, and the projection $p_{0}(a) \in \mathcal{P}_{\mathcal{A}}$ onto the ground space is the ground projection of $a$. This defines a map

$$
p_{0}: A \rightarrow \mathcal{P}_{\mathcal{A}} .
$$

Consider the state space $C_{\mathcal{A}}=\{\rho \in \mathcal{A} \mid \rho \geq 0, \operatorname{tr}(\rho)=1\}$ of positive semi-definite trace-one matrices in $\mathcal{A}$. If $\rho \in C_{\mathcal{A}}$, then the projection onto image $(\rho)$ is called support projection of $\rho$ and will be denoted by $\operatorname{supp}(\rho) \in \mathcal{P}_{\mathcal{A}}$. We call $\rho$ a ground state of $a \in A$ if $\operatorname{supp}(\rho) \leq p_{0}(a)$.

The commutative algebra $\mathcal{A} \subset M_{n}$ of diagonal matrices may be seen as the space of functions

$$
\mathcal{A} \cong \mathbb{C}^{X} \cong\{f: X \rightarrow \mathbb{C}\}
$$

on a configuration space $X$ of cardinality $|X|=n$. A basis of $\mathbb{C}^{X}$ is given by $\left(\delta_{x}\right)_{x \in X}$,

$$
\delta_{x}(y)=\left\{\begin{array}{ll}
0 & \text { if } y \neq x \\
1 & \text { if } y=x
\end{array}, \quad x, y \in X\right.
$$

Hermitian matrices are in one-to-one correspondence with real functions $A=\mathbb{R}^{X}$. The state space $C_{\mathcal{A}}$ of $\mathbb{C}^{X}$ is the simplex of probability distributions on $X$,

$$
\Delta_{X}:=\left\{f: X \rightarrow \mathbb{R} \mid \forall x \in X: f(x) \geqslant 0 \text { and } \sum_{x \in X} f(x)=1\right\} .
$$

The projections are in one-to-one correspondence with 0 -1-functions $\mathcal{P}_{\mathcal{A}} \cong\{0,1\}^{X}$, which we identify with the power set $2^{X}$ of subsets of $X$. The lattice $\mathcal{P}_{\mathcal{A}}$ is a Boolean algebra [1, 13], partially ordered by inclusion. For $p, q \in \mathcal{P}_{\mathcal{A}}$, the complementary projection $p^{\prime}=\mathbb{1}-p$ of $p$ is the set-theoretical complement $p^{\prime}=X \backslash p$, the infimum of $p$ and $q$ is the intersection $p \wedge q=p \cap q$ and the supremum is the union $p \vee q=p \cup q$. While ground spaces are vector spaces, it should be save to call the finite set

$$
p_{0}(f)=\operatorname{argmin}\{f(x) \mid x \in X\} \subset X
$$

ground space of $f \in A$. We refer to $x \in p_{0}(f)$ as a ground configuration of $f$.

We return to an arbitrary ${ }^{*}$-subalgebra $\mathcal{A} \subset M_{n}$ with $\mathbb{1} \in \mathcal{A}$. Opposed to the simplex, $C_{\mathcal{A}}$ has no intuitive visualization in the three-dimensional space [12]. The geometry of $C_{\mathcal{A}}$ is easily grasped by the lattice isomorphism

$$
\phi=\phi_{\mathcal{A}}: \mathcal{P}_{\mathcal{A}} \rightarrow \mathcal{E}\left(C_{\mathcal{A}}\right), \quad p \mapsto\left\{\rho \in C_{\mathcal{A}} \mid \operatorname{supp}(\rho) \leq p\right\},
$$

from projections to exposed faces, see for example Corollary 3.36 of [2]. With notation from (2.1) and (3.2), the projections may be taken to be ground projections, we have

$$
F_{C}=\phi_{\mathcal{A}} \circ p_{0} .
$$


To study normal cones we recall that the relative interior of $F_{C}(a)$ is

$$
\text { ri } F_{C}(a)=\left\{\rho \in C \mid \operatorname{supp}(\rho)=p_{0}(a)\right\}, \quad a \in A .
$$

See Proposition 2.9 of 54 for a proof of $(3.6)$ and (3.7).

Like exposed faces, the normal cones of $C_{\mathcal{A}}$ have a simple algebraic representation. Proposition 2.11 of [54] shows

$$
N_{C}(\rho)=\left\{a \in A \mid \operatorname{supp}(\rho) \leq p_{0}(a)\right\}, \quad \rho \in C_{\mathcal{A}} .
$$

The normal cone at a convex subset being equal to the normal cone at any relative interior point of the convex set, the preceding equation and (3.7) show

$$
N_{C}\left(F_{C}(a)\right)=\left\{b \in A \mid p_{0}(a) \leq p_{0}(b)\right\}, \quad a \in A .
$$

We observe that $C_{\mathcal{A}}$ is not a singleton if $\mathcal{A} \neq \mathbb{C}$. In that case we obtain, combining (3.5) and (2.6), the antitone lattice isomorphism

$$
\nu: \mathcal{P}_{\mathcal{A}} \rightarrow \mathcal{N}_{C_{\mathcal{A}}}, \quad p \mapsto N_{C_{\mathcal{A}}} \circ \phi_{\mathcal{A}}(p) .
$$

Notice that, since $p_{0}: A \rightarrow \mathcal{P}_{\mathcal{A}}$ is onto $\mathcal{P}_{\mathcal{A}} \backslash\{0\}$, the equations $(3.6)$ and $(3.8)$ show

$$
\nu(p)=\left\{a \in A \mid p \leq p_{0}(a)\right\}, \quad p \in \mathcal{P}_{\mathcal{A}} .
$$

\section{Geometric Representations of Ground projections}

We discuss the lattice of ground projections of a linear subspace of hermitian matrices. We recall that the lattice is isomorphic to the lattices of exposed faces and normal cones of a linear image of the state space. We point out the fundamental property that the lattice of ground projections of a linear subspace is coatomistic.

Here and subsequently, let $U \subset A$ a linear subspace and $\pi: A \rightarrow A$ the orthogonal projection onto $U$. The isotone lattice isomorphism (3.5) restricts to another useful map. Let

$$
\mathcal{P}(U):=\left\{p_{0}(u) \mid u \in U\right\} \cup\{0\}
$$

denote the set of ground projections of $U$. The identity $(3.6)$ shows that $\phi$ restricts to the bijection

$$
\left.\phi\right|_{\mathcal{P}(U)}: \mathcal{P}(U) \rightarrow \mathcal{L}
$$

onto the lattice of pre-images of exposed faces of $\pi\left(C_{\mathcal{A}}\right)$, introduced in $(2.2)$. Since $\mathcal{L}$ is a complete lattice whose infimum is the intersection, it follows that $\left.\phi\right|_{\mathcal{P}(U)}$ is an isotone lattice isomorphism and that $\mathcal{P}(U)$, partially ordered by the Löwner partial ordering, is a complete lattice whose infimum is given in terms of intersection of images (3.1). In the classical case $(3.3)$ of $\mathcal{A} \cong \mathbb{C}^{X}$, the infimum in $\mathcal{P}(U)$ is simply the intersection of subsets of $X$. We summarize.

Lemma 4.1. Let $U \subset A$ be a linear subspace. Then $\mathcal{P}(U)$ is a complete lattice. The infimum of $p, q \in \mathcal{P}(U)$ is the projection $r \in \mathcal{P}_{\mathcal{A}}$ whose image is the intersection image $(r)=\operatorname{image}(p) \cap \operatorname{image}(q)$. In the classical case (3.3) we have $p \wedge q=p \cap q$.

There are two important lattice isomorphisms connecting $\mathcal{P}(U)$ to the projection $\pi\left(C_{\mathcal{A}}\right)$ of the state space $C_{\mathcal{A}}$ onto $U$. The isomorphisms 4.1 and 2.4 show that

$$
\left.\pi \circ \phi\right|_{\mathcal{P}(U)}: \mathcal{P}(U) \rightarrow \mathcal{E}(\pi(C))
$$

is an isotone lattice isomorphism [57]. If $\pi(C)$ is not a singleton, then by (2.6)

$$
\left.N_{\pi(C)} \circ \pi \circ \phi\right|_{\mathcal{P}(U)}: \mathcal{P}(U) \rightarrow \mathcal{N}(\pi(C))
$$




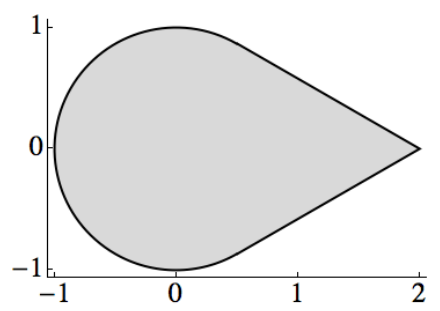

FiguRE 1. The convex hull of the unit disk and the point $(2,0)$ has two boundary segments containing only one atom, $\{(2,0)\}$, of the lattice of exposed faces. They cannot be written as suprema of atoms.

is an antitone lattice isomorphism. Using (2.7) and the map $\nu$ from 3.9 , we notice

$$
N_{\pi(C)} \circ \pi \circ \phi(p)=\nu(p) \cap U, \quad p \in \mathcal{P}_{\mathcal{A}},
$$

where $\nu(p)$ is a normal cone of the state space $C_{\mathcal{A}}$.

Remark 4.2. The convex set $\pi\left(C_{\mathcal{A}}\right)$ is affinely isomorphic to convex sets studied in other fields: Algebraic polar of a spectrahedron [47, joint algebraic numerical range [42], and state space of an operator system [46]. See [57] for details about the latter.

The lattice of exposed faces $\mathcal{E}\left(\pi\left(C_{\mathcal{A}}\right)\right)$ is generated by certain maximal elements.

Definition 4.3 (Atoms and coatoms). Let $\mathcal{K}$ be a complete lattice with smallest element 0 and greatest element 1 . We say 0 and 1 are improper elements of $\mathcal{K}$ and we call $x \in \mathcal{K}$ a proper element of $\mathcal{K}$ if and only $x \notin\{0,1\}$. An atom of $\mathcal{K}$ is a minimal element of $\mathcal{K} \backslash\{0\}$. The lattice $\mathcal{K}$ is atomistic if each element is the supremum of the atoms which it contains. A coatom of $\mathcal{K}$ is a maximal element of $\mathcal{K} \backslash\{1\}$. The lattice $\mathcal{K}$ is coatomistic if each element is the infimum of the coatoms in which it is contained.

The lattice $\mathcal{E}\left(\pi\left(C_{\mathcal{A}}\right)\right)$ is coatomistic [55] and so is $\mathcal{P}(U)$ by virtue of [4.2, as was shown in [57].

Lemma 4.4. Let $U \subset A$ be a linear subspace. Then the lattice $\mathcal{P}(U)$ is coatomistic.

The lattice $\mathcal{E}(\pi(C))$ is generally not atomistic. An example with $n=3$ is depicted in Figure 1 in coordinates $\left\{\left(\left\langle\rho, a_{1}\right\rangle,\left\langle\rho, a_{2}\right\rangle\right) \mid \rho \in C_{M_{3}}\right\} \subset \mathbb{R}^{2}$ where $U$ is the span of

$$
a_{1}=\left(\begin{array}{lll}
0 & 1 & 0 \\
1 & 0 & 0 \\
0 & 0 & 2
\end{array}\right) \text { and } a_{2}=\left(\begin{array}{ccc}
0 & -\mathrm{i} & 0 \\
\mathrm{i} & 0 & 0 \\
0 & 0 & 0
\end{array}\right) \text {. }
$$

In the classical case 3.3 the state space $C_{\mathcal{A}}=\Delta_{X}$ is a simplex, $\pi\left(\Delta_{X}\right)$ a polytope, and $\mathcal{E}\left(\pi\left(\Delta_{X}\right)\right)$ atomistic 2 . The atoms are the exposed points and the coatoms the facets of $\pi\left(\Delta_{X}\right)$, that is exposed faces of codimension one, see Theorem 2.7 of [62].

Remark 4.5 (Top to bottom via minors). If $\mathbb{1} \in U$ then every non-zero element of $\mathcal{P}(U)$ is the ground projection of a positive semi-definite $u \in U$ with $p_{0}(u)=\operatorname{ker}(u)$. The Sylvester criterion [32] shows for $k=1, \ldots, n$ that $\{p \in \mathcal{P}(U) \mid \operatorname{rk}(p) \geqslant k\}$ is formed by the ground projections of solutions of the real algebraic system

$\{u \in U \mid$ the minors of $u$ of size $n-k+1$ are zero and the principal minors of $u$ of size at most $n-k$ are non-negative $\}$.

Lemma 4.4 suggests to solve the algebraic systems by decreasing order of the rank $k=n-1, n-2, \ldots, 1$. This order has also the advantage to start with the system of the smallest algebraic degree.

\footnotetext{
${ }^{2}$ The term (co-) atomistic employed here is called (co-) atomic in [62].
} 


\section{A VARIATION PRINCIPLE FOR GROUND SPACES}

The variation principle of Proposition 2.6 is applied here to ground projections.

Lemma 5.1. Let $U \subset A$ be a linear subspace such that $\pi(C)$ is no singleton, and let $p \in \mathcal{P}_{\mathcal{A}}$. Then $p \in \mathcal{P}(U)$ holds if and only if $p$ is the greatest element of $\left\{q \in \mathcal{P}_{\mathcal{A}} \mid\right.$ $\nu(q) \cap U=\nu(p) \cap U\}$ in the Löwner partial ordering.

Proof: Proposition 2.6 shows for $F \in \mathcal{E}(C)$ that $F \in \mathcal{L}$ if and only if $F$ is the greatest element of

$$
\left\{G \in \mathcal{E}(C) \mid N_{C}(G) \cap U=N_{C}(F) \cap U\right\} .
$$

The lattice isomorphisms $\phi: \mathcal{P}_{\mathcal{A}} \rightarrow \mathcal{E}(C)$ from $(3.5)$ and $\left.\phi\right|_{\mathcal{P}(U)}: \mathcal{P}(U) \rightarrow \mathcal{L}$ from (4.1) show for $p \in \mathcal{P}_{\mathcal{A}}$ that $p \in \mathcal{P}(U)$ if and only if $p$ is the greatest element of

$$
\left\{q \in \mathcal{P}_{\mathcal{A}} \mid N_{C} \circ \phi(q) \cap U=N_{C} \circ \phi(p) \cap U\right\} .
$$

The definition $(3.9)$ of $\nu$ finishes the proof.

To employ Lemma 5.1 algebraically, we use 3.10 and write

$$
\nu(p)=\left\{a \in A \mid p \leq p_{0}(a)\right\}=\mathbb{R} \mathbb{1}+p^{\prime} \mathcal{A}^{+} p^{\prime}, \quad p \in \mathcal{P}_{\mathcal{A}} .
$$

Recall from 4.4 that $\nu(p) \cap U=\left(\mathbb{R} \mathbb{1}+p^{\prime} \mathcal{A}^{+} p^{\prime}\right) \cap U$ is a normal cone of $\pi(C)$.

Definition 5.2. For all $p \in \mathcal{P}_{\mathcal{A}}$, let $K(p):=p^{\prime} \mathcal{A}^{+} p^{\prime} \cap U$.

The substitution of $\nu(p) \cap U$ with $K(p)$ is not always faithful. For example, let $\mathcal{A}=M_{2}$, let $U$ be the span of $X=\left(\begin{array}{ll}0 & 1 \\ 1 & 0\end{array}\right)$ and $Y=\left(\begin{array}{cc}0 & -\mathrm{i} \\ \mathrm{i} & 0\end{array}\right)$, and let $u \in U$ have spectral norm one. Then $p_{0}(u)=\frac{1}{2}(\mathbb{1}-u)$ is the ground projection of $u$ and

$$
\nu\left(p_{0}(u)\right) \cap U=\{\lambda \cdot u \mid \lambda \geqslant 0\},
$$

while $K(p)=\{0\}$ for all $p \in \mathcal{P}_{\mathcal{A}}$.

Lemma 5.3. Let $U \subset A$ be a linear subspace with $U \supsetneq \mathbb{R} \mathbb{1}$. Then for all $p, q \in \mathcal{P}_{\mathcal{A}}$ we have $\nu(p) \cap U=\nu(q) \cap U \Longleftrightarrow K(p)=K(q)$.

Proof: We prove " $\Leftarrow "$. We have for all $p \in \mathcal{P}_{\mathcal{A}}$

$$
\nu(p) \cap U=\left(\mathbb{R} \mathbb{1}+p^{\prime} \mathcal{A}^{+} p^{\prime}\right) \cap U=\mathbb{R} \mathbb{1}+\left(p^{\prime} \mathcal{A}^{+} p^{\prime} \cap U\right)=\mathbb{R} \mathbb{1}+K(p) .
$$

The first equality of $(5.2)$ holds by (5.1), the second equality requires $\mathbb{1} \in U$, see equation (22) of [55]. We prove " $\Rightarrow$ ". Unless stated otherwise, $\mathbb{1} \in U$ is not assumed in the sequel. For $p \in \mathcal{P}_{\mathcal{A}}$ we have

$$
K(p)=\{u \in U \mid u \geq 0, p u=0\} .
$$

Let $p \neq 0$. Then for any $a \in A$ such that $p a=0$, each of the statements $a \geq 0$ or $p \leq p_{0}(a)$ implies $\operatorname{ker}(a)=p_{0}(a)$. Hence

$$
K(p)=\left\{u \in U \mid p \leq p_{0}(u), p u=0\right\}, \quad p \neq 0,
$$

that is by 5.1

$$
K(p)=\{u \in \nu(p) \cap U \mid p u=0\}, \quad p \neq 0 .
$$

To finish the proof it suffices to show that $\nu(0) \cap U \neq \nu(p) \cap U$. We have

$$
\nu(0) \cap U=N_{C}(\varnothing) \cap U=A \cap U=U=N_{\pi(C)}(\varnothing),
$$

and by 4.4 and 2.5

$$
\nu(p) \cap U=N_{\pi(C)}(\pi \circ \phi(p))=N_{\pi(C)}\left(\operatorname{cl}_{\mathcal{E}(\pi(C))}(\pi \circ \phi(p))\right.
$$


holds. Since $p \neq 0$, the exposed face $\mathrm{cl}_{\mathcal{E}(\pi(C))}(\pi \circ \phi(p))$ is non-empty. Assuming $\pi(C)$ is not a singleton, which is implied by $U \supsetneq \mathbb{R} \mathbb{1}$, the map $N_{\pi(C)}: \mathcal{E}(\pi(C)) \rightarrow \mathcal{N}(\pi(C))$ is the isomorphism (2.6). This completes the proof.

Notice that the statement of Lemma 5.3 is wrong for $U=\mathbb{R} \mathbb{1}$. Here $\nu(p) \cap U=U$ for all $p \in \mathcal{P}_{\mathcal{A}}$, while $K(0)=\{\lambda \mathbb{1} \mid \lambda \geqslant 0\}$ and $K(p)=\{0\}$ for all $p \in \mathcal{P}_{\mathcal{A}} \backslash\{0\}$.

Theorem 5.4. Let $U \subset A$ be a linear subspace with $\mathbb{1} \in U$ and let $p \in \mathcal{P}_{\mathcal{A}}$. Then $p \in \mathcal{P}(U)$ holds if and only if $p$ is the greatest element of $\left\{q \in \mathcal{P}_{\mathcal{A}} \mid K(q)=K(p)\right\}$ in the Löwner partial ordering.

Proof: The case $U=\mathbb{R} \mathbb{1}$ is verified in the preceding paragraph. If $U \supsetneq \mathbb{R} \mathbb{1}$ then the claim follows from Lemmas 5.1 and 5.3 .

Using the linear spaces $L(p):=p^{\prime} A p^{\prime} \cap U, p \in \mathcal{P}_{\mathcal{A}}$, may simplify matters.

Lemma 5.5. Let $U \subset A$ be a linear subspace, let $p \in \mathcal{P}_{\mathcal{A}}$. Then $K(p)=L(p) \cap \mathcal{A}^{+}$. In the classical case (3.3) we have $L(p)=\left(p A p+U^{\perp}\right)^{\perp}$.

\section{Contoms of the lattice of Ground spaces}

Here we characterize the coatoms of the lattice of ground projections $\mathcal{P}(U)$.

Theorem 6.1. Let $U \subset A$ be a linear subspace with $\mathbb{1} \in U$ and let $p \in \mathcal{P}(U)$.

(1) The projection $p$ is a coatom of $\mathcal{P}(U)$ if and only if $K(p)$ is a ray.

(2) Let $p \neq 0$ and $d=\operatorname{dim} K(p)$. There are coatoms $q_{1}, \ldots, q_{d}$ of $\mathcal{P}(U)$ such that $p=q_{1} \wedge \ldots \wedge q_{d}$ and such that the rays $K\left(q_{1}\right), \ldots, K\left(q_{d}\right)$ are linearly independent exposed extreme rays of $K(p)$.

Proof: Let $U=\mathbb{R} \mathbb{1}$. Then $\mathcal{P}(U)=\{0, \mathbb{1}\}$ and indeed $K(0)=\{\lambda \mathbb{1} \mid \lambda \geqslant 0\}$ is a ray while $K(\mathbb{1})=\{0\}$. The second assertion is true as the infimum of $\varnothing$ is $\mathbb{1}$.

In the following we assume $U \supsetneq \mathbb{R} \mathbb{1}$. Let $U_{0} \subset U$ be the space of traceless matrices in $U$ and notice $\mathcal{P}\left(U_{0}\right)=\mathcal{P}(U)$. The convex set $\pi_{U_{0}}(C)$ is a proper convex set in the sense of [57], that is $\pi_{U_{0}}(C)$ has non-empty interior in $U_{0}$ and $\mathcal{E}\left(\pi_{U_{0}}(C)\right)$ contains a proper exposed face. Under these (technical) assumptions, Theorem 6.2 and Corollary 6.3 of [57] show that any non-empty face of any normal cone $N$ of $\pi_{U_{0}}(C)$ lies in $\mathcal{N}_{\pi_{U_{0}}(C)}$. Hence, Remark 2.2(4) of [57] shows that $N$ has $\operatorname{dim}(N)$ linearly independent exposed rays which lie in $\mathcal{N}_{\pi_{U_{0}}(C)}$. These properties of $\pi_{U_{0}}(C)$ are used in the sequel without further mention.

Since $\nu(p) \pm \mathbb{R} \mathbb{1} \subset \nu(p)$ holds, we have $\mathbb{R} \mathbb{1}+\left(U_{0} \cap \nu(p)\right)=\left(\mathbb{R} \mathbb{1}+U_{0}\right) \cap \nu(p)$ by Lemma 5.1 of [55]. Hence

$$
\nu(p) \cap U=\mathbb{R} \mathbb{1}+\left(\nu(p) \cap U_{0}\right), \quad p \in \mathcal{P}_{\mathcal{A}} .
$$

Notice that the right-hand side of $(6.1)$ is the direct sum of a line and a convex cone, the cone being pointed if $p \neq 0$. By (5.2) we have

$$
\nu(p) \cap U=\mathbb{R} \mathbb{1}+K(p), \quad p \in \mathcal{P}_{\mathcal{A}} .
$$

Notice that the right-hand side of 6.2 is the sum of a line and a pointed convex cone, the sum being direct if $p \neq 0$.

Proof of (1). The antitone lattice isomorphism $\mathcal{P}\left(U_{0}\right) \rightarrow \mathcal{N}\left(\pi_{U_{0}}(C)\right)$ defined in (4.3) is the function $p \mapsto \nu(p) \cap U_{0}$, as observed in (4.4). Hence, a projection $p \in \mathcal{P}\left(U_{0}\right)$ is a coatom of $\mathcal{P}\left(U_{0}\right)$ if and only if $\nu(p) \cap U_{0}$ is an atom of $\mathcal{N}\left(\pi_{U_{0}}(C)\right)$. 
Theorem 3.2 of [57] shows that the atoms of $\mathcal{N}\left(\pi_{U_{0}}(C)\right)$ are the rays in $\mathcal{N}\left(\pi_{U_{0}}(C)\right)$. If $p \neq 0$ then by (6.1) and $(6.2)$ the cone $\nu(p) \cap U_{0}$ is a ray if and only if $K(p)$ is a ray. This proves the claim for $p \neq 0$. The cone $K(0)$ is not a ray, as $U \supsetneq \mathbb{R} \mathbb{1}$ and as $\mathbb{1}$ is an interior point of $\mathcal{A}^{+}$. In agreement with that, 0 is not a coatom of $\mathcal{P}\left(U_{0}\right)$, because $\mathcal{P}\left(U_{0}\right)$, has a proper element by $\left.\sqrt{4.2}\right)$, as $\pi_{U_{0}}(C)$ has a proper exposed face.

Proof of (2). Let $F$ be a proper exposed face of $\pi_{U_{0}}(C)$ and $d=\operatorname{dim}\left(N_{\pi_{U_{0}}(C)}(F)\right)$. Corollary 2.3 of [57] shows that there are coatoms $F_{1}, \ldots, F_{d}$ of $\mathcal{E}\left(\pi_{U_{0}}(C)\right)$ such that $F=F_{1} \cap \ldots \cap F_{d}$ and such that the rays $N_{\pi_{U_{0}}(C)}\left(F_{i}\right), i=1, \ldots, d$, are linearly independent exposed extreme rays of $N_{\pi_{U_{0}}(C)}(F)$. An analogous statement concerning proper ground projections $p$ of $U_{0}$ follows from the lattice isomorphism 4.2,

$$
\left.\pi_{U_{0}} \circ \phi\right|_{\mathcal{P}\left(U_{0}\right)}: \mathcal{P}\left(U_{0}\right) \rightarrow \mathcal{E}\left(\pi_{U_{0}}(C)\right) .
$$

Let $N=N_{\pi_{U_{0}}(C)}\left(\pi_{U_{0}} \circ \phi(p)\right)$ and $d=\operatorname{dim}(N)$. There are coatoms $q_{1}, \ldots, q_{d}$ of $\mathcal{P}\left(U_{0}\right)$ such that $p=q_{1} \wedge \ldots \wedge q_{d}$ and such that the rays $r_{i}=N_{\pi_{U_{0}}(C)}\left(\pi_{U_{0}} \circ \phi\left(q_{i}\right)\right)$, $i=1, \ldots, d$, are linearly independent exposed extreme rays of $N$. Using (4.4), we get $N=\nu(p) \cap U_{0}$ and $r_{i}=\nu\left(q_{i}\right) \cap U_{0}, i=1, \ldots, d$. From 6.1 and 6.2 follows that $d=\operatorname{dim}\left(\nu(p) \cap U_{0}\right)=\operatorname{dim} K(p)$. To see that the rays $K\left(q_{i}\right), i=1, \ldots, d$, are exposed rays of $K(p)$, we notice from (6.1) that $r_{i}=\nu\left(q_{i}\right) \cap U_{0}$ defines an exposed half-plane $\mathbb{R} \mathbb{1}+r_{i}=\nu\left(q_{i}\right) \cap U$ of $\mathbb{R} \mathbb{1}+N=\nu(p) \cap U$. The linear space $V=\{a \in A \mid p a=0\}$ intersects $\nu(p) \cap U$ transversally to its lineality space $\mathbb{R} \mathbb{1}$ and the intersection is the pointed convex cone $K(p)$ by $(5.3)$. Hence $\left(\mathbb{R} \mathbb{1}+r_{i}\right) \cap V$ is an exposed ray of $K(p)$. Since $q_{i} \geq p$ we have $\left(\mathbb{R} \mathbb{1}+r_{i}\right) \cap V=K\left(q_{i}\right)$. Adding and subtracting lineality also shows that the linear independence of $\left\{r_{i}\right\}_{i=1}^{d}$ implies that of $\left\{K\left(q_{i}\right)\right\}_{i=1}^{d}$. This proves the claim for proper $p$. The assertion is trivial for $p=\mathbb{1}$. $\square$

\section{A Simple NON-COMMUtative EXAMPle}

We compute ground spaces of the example (4.5) from Section 4 and compare with results concerning coatoms from Section 6 .

Let $\sigma_{X}=\left(\begin{array}{ll}0 & 1 \\ 1 & 0\end{array}\right)$ and $\sigma_{Y}=\left(\begin{array}{cc}0 & -\mathrm{i} \\ \mathrm{i} & 0\end{array}\right)$. The space $U$ is the real span of

$$
\mathbb{1}, \quad\left(\begin{array}{lll}
0 & 1 & 0 \\
1 & 0 & 0 \\
0 & 0 & 2
\end{array}\right)=\sigma_{X} \oplus 2, \quad\left(\begin{array}{ccc}
0 & -\mathrm{i} & 0 \\
\mathrm{i} & 0 & 0 \\
0 & 0 & 0
\end{array}\right)=\sigma_{Y} \oplus 0 \quad \in M_{3} .
$$

We write $u \in U$ in the form

$$
u=\lambda \mathbb{1}+\operatorname{Re}(z)\left(\sigma_{X} \oplus 2\right)+\operatorname{Im}(z) \sigma_{Y} \oplus 0, \quad \lambda \in \mathbb{R}, z \in \mathbb{C} .
$$

The ground space of $u$ is independent of $\lambda$ and invariant under scaling of $u$ with positive scalars. We assume $|z|=1$. Using rank-one projections $p(z):=\frac{1}{2}\left(\begin{array}{ll}1 & \bar{z} \\ z & 1\end{array}\right)$,

$$
u=[(\lambda-1) p(-z)+(\lambda+1) p(z)] \oplus[\lambda+2 \operatorname{Re}(z)]
$$

is the spectral decomposition of $u$, the eigenvalues being $\{\lambda \pm 1, \lambda+2 \operatorname{Re}(z)\}$.

Case a), $\operatorname{Re}(z)=-1 / 2$. Let $z_{ \pm}:=-\frac{1}{2} \pm \mathrm{i} \frac{\sqrt{3}}{2}$ and $u_{ \pm}:=2 p\left(z_{ \pm}\right) \oplus 0$. For $\lambda=1$ and $z=z_{ \pm}$we obtain $u=u_{ \pm}$in equation (7.1), and $p_{0}(u)$ is the rank-two coatom

$$
p_{ \pm}:=p_{0}\left(u_{ \pm}\right)=p\left(-z_{ \pm}\right) \oplus 1
$$

of $\mathcal{P}(U)$. The cone

$$
K\left(p_{ \pm}\right)=\left\{u \in U \mid u \geq 0, p\left(-z_{ \pm}\right) \oplus 1 \leq \operatorname{ker}(u)\right\}=\left\{\eta \cdot u_{ \pm} \mid \eta \geqslant 0\right\}
$$

is a ray in accord with Theorem 6.1(1). 
Case b), $\operatorname{Re}(z)>-1 / 2$. Taking $\lambda=1$, we have $u=2 p(z) \oplus(1+2 \operatorname{Re}(z))$ in equation (7.1), and $p_{0}(u)=p(-z) \oplus 0$. Since $p_{0}(u) \nless p_{ \pm}$and since $p_{ \pm}$are the only rank-two elements of $\mathcal{P}(U)$, the projection $p_{0}(u)$ is a coatom of $\mathcal{P}(U)$. The cone

$$
\begin{aligned}
K\left(p_{0}(u)\right) & =\{u \in U \mid u \geq 0, p(-z) \oplus 0 \leq \operatorname{ker}(u)\} \\
& =\{\eta \cdot(2 p(z) \oplus(1+\operatorname{Re} z)) \mid \eta \geqslant 0\}
\end{aligned}
$$

is a ray in agreement with Theorem 6.1(1).

Case c), $\operatorname{Re}(z)<-1 / 2$. Equation (7.1) shows $p_{0}(u)=0 \oplus 1=p_{+} \wedge p_{-}$. The cone

$$
\begin{aligned}
K(0 \oplus 1) & =\{u \in U \mid u \geq 0,0 \oplus 1 \leq \operatorname{ker}(u)\} \\
& =\{\eta \cdot[(-2 \operatorname{Re} \tilde{z}-1) p(-\tilde{z})+(-2 \operatorname{Re} \tilde{z}+1) p(\tilde{z})] \mid \\
& \quad|\tilde{z}|=1, \operatorname{Re}(\tilde{z}) \leqslant-1 / 2, \eta \geqslant 0\} \oplus 0 \\
& =\left\{\operatorname{Re}(\tilde{z})\left(\sigma_{X}-2 \cdot \mathbb{1}\right)+\operatorname{Im}(\tilde{z}) \sigma_{Y} \mid \arg (\tilde{z}) \in\left[\frac{2}{3} \pi, \frac{4}{3} \pi\right]\right\} \oplus 0
\end{aligned}
$$

has dimension two. Since $\arg \left(z_{+}\right)=\frac{2}{3} \pi$ and $\arg \left(z_{-}\right)=\frac{4}{3} \pi$, we have

$$
\operatorname{Re}\left(z_{ \pm}\right)\left(\sigma_{X}-2 \cdot \mathbb{1}\right)+\operatorname{Im}\left(z_{ \pm}\right) \sigma_{Y}=\mathbb{1}+\left(\begin{array}{cc}
0 & \bar{z}_{ \pm} \\
z_{ \pm} & 0
\end{array}\right)=2 p\left(z_{ \pm}\right)=u_{ \pm} .
$$

So $K\left(p_{ \pm}\right)$are the extreme rays of $K(0 \oplus 1)$ in agreement with Theorem 6.1(2).

\section{MANY-BODY SYSTEMS}

In this section we discuss $k$-local Hamiltonians and quantum marginals.

We consider a composite system of $N \in \mathbb{N}$ units, labeled by $[N]:=\{1, \ldots, N\}$. For each unit $i \in[N]$ we choose a Hilbert space $\mathbb{C}^{n_{i}}, n_{i} \in \mathbb{N}$, and a ${ }^{*}$-algebra $\mathcal{A}_{i}$ acting on $\mathbb{C}^{n_{i}}$ and containing the $n_{i}$-by- $n_{i}$ identity matrix. For any subset $\nu \subset[N]$, the tensor product $\bigotimes_{i \in \nu} \mathbb{C}^{n_{i}}$ is the Hilbert space and $\mathcal{A}_{\nu}:=\bigotimes_{i \in \nu} \mathcal{A}_{i}$ is the algebra, with identity denoted by $\mathbb{1}_{\nu}$, of the system composed of the units in $\nu$. The full system has algebra

$$
\mathcal{A}:=\mathcal{A}_{[N]}=\bigotimes_{i \in[N]} \mathcal{A}_{i}
$$

By definition, a $k$-local Hamiltonian is of the form

$$
a=\sum_{|\nu|=k} a(\nu) \otimes \mathbb{1}_{\nu^{\prime}},
$$

where $\nu^{\prime}=[N] \backslash \nu$, where $a(\nu) \in \mathcal{A}_{\nu}$ is a hermitian matrix, and where the sum extends over subsets $\nu \subset[N]$ with $|\nu|=k$. We denote the real vector space of $k$-local Hamiltonians by $U_{(k)}$.

From now on we consider $U=U_{(k)}$. We pointed out in the introduction that $\pi(C)$ is isomorphic to the set of $k$-body marginals. To see this, let $\nu \subset[N]$. The partial trace over the subsystem $\nu$ is the linear map $\operatorname{tr}_{\nu}: \mathcal{A} \rightarrow \mathcal{A}_{\nu^{\prime}}$ which is the adjoint of the embedding $\mathcal{A}_{\nu^{\prime}} \hookrightarrow \mathcal{A}, a \mapsto a \otimes \mathbb{1}_{\nu}$ with respect to the Hilbert-Schmidt inner product. If $\rho \in C_{\mathcal{A}}$ is a state on $\mathcal{A}$ then $\operatorname{tr}_{\nu^{\prime}}(\rho) \in C_{\mathcal{A}_{\nu}}$ is the marginal of $\rho$ on subsystem $\nu$. For $k=1, \ldots, N$ we define the linear map

$$
\operatorname{tr}_{(k)}: \mathcal{A} \rightarrow \times_{|\nu|=k} \mathcal{A}_{\nu}, \quad a \mapsto\left(\operatorname{tr}_{\nu^{\prime}}(a)\right)_{|\nu|=k}
$$

to the cartesian product (direct sum) of algebras $\mathcal{A}_{\nu},|\nu|=k$. The set of $k$-body marginals or $k$-body reduced density matrices [16] is

$$
\mathcal{D}_{(k)}:=\left\{\operatorname{tr}_{(k)}(\rho) \mid \rho \in C_{\mathcal{A}}\right\} .
$$

The restricted linear map

$$
\left.\operatorname{tr}_{(k)}\right|_{\pi\left(C_{\mathcal{A}}\right)}: \pi\left(C_{\mathcal{A}}\right) \rightarrow \mathcal{D}_{(k)}
$$

is a bijection because of $\operatorname{tr}_{(k)}=\operatorname{tr}_{(k)} \circ \pi$ and since $\left.\operatorname{tr}_{(k)}\right|_{U_{(k)}}$ is injective. 
The inclusion $\mathcal{D}_{(k)} \subset X_{|\nu|=k} C_{\mathcal{A}_{\nu}}$ is strict for $k \geqslant 2$ as it follows from comparison of dimensions. Let $n_{1}=\cdots=n_{N}$ and $\mathcal{A}_{i}=M_{n_{1}}, i \in[N]$. Then Proposition 1 of [59] and (8.4) show

$$
\operatorname{dim}\left(\mathcal{D}_{(k)}\right)=\operatorname{dim}\left(\pi\left(C_{\mathcal{A}}\right)\right)=\operatorname{dim}\left(U_{(k)}\right)-1=\sum_{\ell=1}^{k}\left(\begin{array}{c}
N \\
\ell
\end{array}\right)\left(n_{1}^{2}-1\right)^{\ell} .
$$

For $k=2$ and three qubits this gives $\operatorname{dim}\left(\mathcal{D}_{(k)}\right)=36<45=\operatorname{dim}\left(C_{M_{2}} \times C_{M_{2}} \times C_{M_{2}}\right)$.

In the classical case (3.3), the algebra of unit $i \in[N]$ is the space of functions $\mathcal{A}_{i}=\mathbb{C}^{X_{i}}$ on a configuration space $X_{i}$ with $\left|X_{i}\right|=n_{i}, i \in[N]$. The algebra $\mathcal{A}_{\nu}=\mathbb{C}^{X_{\nu}}$ is the space of functions on the configuration space of subsystem $\nu$,

$$
X_{\nu}:=\times_{i \in \nu} X_{i}, \quad \nu \subset[N] .
$$

The full configuration space is $X:=X_{[N]}$. Viewing $x \in X$ as a sequence, let $x_{\nu} \in X_{\nu}$ denote its truncation to $\nu \subset[N]$. This means $x=\left(x_{i}\right)_{i \in[N]}$ for $x_{i} \in X_{i}, i \in[N]$, and $x_{\nu}=\left(x_{\nu, i}\right)_{i \in \nu}$ for $x_{\nu, i}=x_{i}, i \in \nu$. Denoting the disjoint union by

$$
\bigcup_{|\nu|=k} X_{\nu}=\left\{(\nu, y):|\nu|=k, y \in X_{\nu}\right\}
$$

the matrix of 8.2 with respect to the bases $\left(\delta_{x}\right)_{x \in X}$ and $\left(\delta_{(\nu, y)}\right)_{(\nu, y) \in \bigcup_{|\nu|=k} X_{\nu}}$ is

$$
\left(\operatorname{tr}_{(k)}\right)_{(\nu, y), x}=\left\{\begin{array}{ll}
0 & \text { if } y \neq x_{\nu} \\
1 & \text { if } y=x_{\nu}
\end{array}, \quad x \in X,|\nu|=k, y \in X_{\nu} .\right.
$$

The simplex $C_{\mathcal{A}}=\Delta_{X}$ is the convex hull of $\left(\delta_{x}\right)_{x \in X}$. Hence $(8.3)$ shows that $\mathcal{D}_{(k)}$ is the convex hull of the columns of the matrix 8.6). The polytope $\mathcal{D}_{(k)}$ is well-known in mathematical statistics [22, 25, 34]. If $n_{1}=\cdots=n_{N}$, then Proposition 1 of [59] and (8.4) show

$$
\operatorname{dim}\left(\mathcal{D}_{(k)}\right)=\operatorname{dim}\left(\pi_{U_{(k)}}\left(\Delta_{X}\right)\right)=\operatorname{dim}\left(U_{(k)}\right)-1=\sum_{\ell=1}^{k}\left(\begin{array}{c}
N \\
\ell
\end{array}\right)\left(n_{1}-1\right)^{\ell} .
$$

\section{A Simple three-Bit EXAMPle}

We discuss ground spaces of 2-local 3-bit Hamiltonians.

The three-bit configuration space (8.5) is $X=\{0,1\} \times\{0,1\} \times\{0,1\}$. The function

$$
f: X \rightarrow \mathbb{R}, \quad f\left(x_{1}, x_{2}, x_{3}\right)=(-1)^{x_{1}+x_{2}+x_{3}}
$$

spans the complement of $U_{(2)}$ in $A=\mathbb{R}^{X}$, as $\operatorname{dim}\left(U_{(2)}\right)=7$ by 8.7). Let $p \in \mathcal{P}_{\mathcal{A}}$. Lemma 5.5 shows $K(p)=\{u \in L(p) \mid u \geq 0\}$ where

$$
L(p)=p^{\prime} A p^{\prime} \cap U_{(2)}=(p A p+\mathbb{R} \cdot f)^{\perp} .
$$

Let $|p|=7$. We prove $p \notin \mathcal{P}\left(U_{(2)}\right)$. Since $f$ has full rank and $p^{\prime}$ only one non-zero coefficient, for all $a \in p^{\prime} A p^{\prime}$ the statement $\langle a, f\rangle=0$ is equivalent to $a=0$. This proves $K(p)=\{0\}$. Theorem 5.4 shows $p \notin \mathcal{P}\left(U_{(2)}\right)$ because $K(\mathbb{1})=\{0\}$.

Let $|p| \leqslant 5$. We prove that $p$ is not a coatom of $\mathcal{P}\left(U_{(2)}\right)$. First, let us assume that for all configurations $x \in p^{\prime}$ we have $f(x)=1$ (the case $f(x)=-1$ is analogous). A diagonal matrix $a \in A$ lies in $K(p)$ if and only if $a \in p^{\prime} A p^{\prime}, a \geq 0$, and $a \in U$. Assuming the latter, we obtain $0=\langle a, f\rangle=\operatorname{tr}(a)$ which implies $a=0$. This proves $K(p)=\{0\}$ and shows $p \notin \mathcal{P}\left(U_{(2)}\right)$ as in the preceding paragraph. Second, assume without loss of generality that there are $x, y \in p^{\prime}$ such that $f(x)=1$ and $f(y)=-1$. Since $\left|p^{\prime}\right| \geqslant 3$, there is $z \in p^{\prime} \backslash\{x, y\}$ such that $f(z)=-1$ (the case $f(z)=1$ is analogous). Using the basis (3.4) we obtain

$$
\delta_{x}+\delta_{y}, \delta_{x}+\delta_{z} \in K(p)
$$

which shows $\operatorname{dim} K(p)>1$. Theorem 6.1(1) shows that $p$ is not a coatom of $\mathcal{P}\left(U_{(2)}\right)$. 

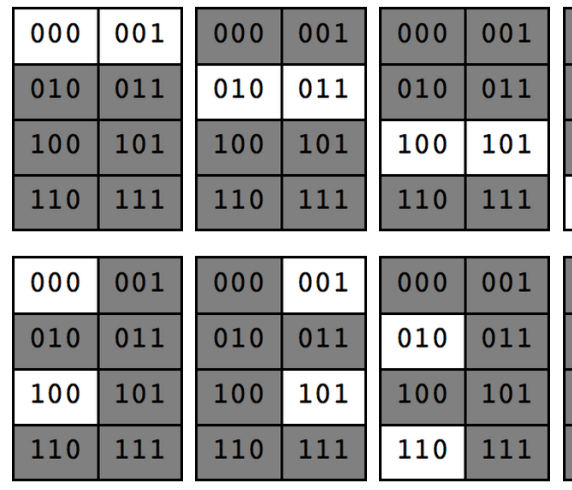

\begin{tabular}{|l|l|}
\hline 000 & 001 \\
\hline 010 & 011 \\
\hline 100 & 101 \\
\hline 110 & 111 \\
\hline 000 & 001 \\
\hline 010 & 011 \\
\hline 100 & 101 \\
\hline 110 & 111 \\
\hline
\end{tabular}

\begin{tabular}{|l|l|}
\hline 000 & 001 \\
\hline 010 & 011 \\
\hline 100 & 101 \\
\hline 110 & 111 \\
\hline 000 & 001 \\
\hline 010 & 011 \\
\hline 100 & 101 \\
\hline 110 & 111 \\
\hline
\end{tabular}

\begin{tabular}{|l|l|}
\hline 000 & 001 \\
\hline 010 & 011 \\
\hline 100 & 101 \\
\hline 110 & 111 \\
\hline 000 & 001 \\
\hline 010 & 011 \\
\hline 100 & 101 \\
\hline 110 & 111 \\
\hline
\end{tabular}

\begin{tabular}{|l|l|}
\hline 000 & 001 \\
\hline 010 & 011 \\
\hline 100 & 101 \\
\hline 110 & 111 \\
\hline 000 & 001 \\
\hline 010 & 011 \\
\hline 100 & 101 \\
\hline 110 & 111 \\
\hline
\end{tabular}

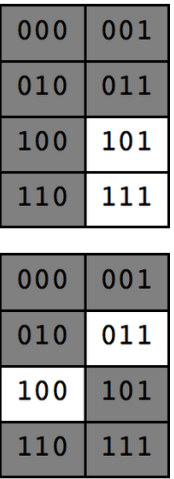

Figure 2. The tables depict the 16 coatoms of $\mathcal{P}\left(U_{(2)}\right)$. Ground configurations are distinguished by a dark background.

Let $|p|=6$ and let $p^{\prime}=\{x, y\}$ for distinct $x, y \in X$. If $f(x)=f(y)$ then $K(p)=\{0\}$ follows as in the case $|p| \leqslant 5$ treated above. If $f(x) \neq f(y)$ then $a \in p^{\prime} A p^{\prime}$ and $a \in U$ imply that $a$ is a scalar multiple of $\delta_{x}+\delta_{y}$, so

$$
K(p)=\{u \in U \mid u \geq 0, p \leq \operatorname{ker}(u)\}=\left\{\eta \cdot\left(\delta_{x}+\delta_{y}\right) \mid \eta \geqslant 0\right\} .
$$

This shows that $p$ is a coatom of $\mathcal{P}\left(U_{(2)}\right)$. See Figure 2 for drawings.

The complete bipartite graph $K_{4,4}$ with vertex set $X$ and bi-partition

$$
V_{i}=\{x \in X \mid f(x)=i\}, \quad i= \pm 1,
$$

allows to have further insights into the lattice $\mathcal{P}\left(U_{(2)}\right)$, for three bits. We showed that $p \subset X$ is a coatom of $\mathcal{P}\left(U_{(2)}\right)$ if and only if $p^{\prime}$ is an edge of $K_{4,4}$. Dually, $p \subset X$ is an atom of the dual lattice

$$
\mathcal{P}\left(U_{(2)}\right)^{*}=\left\{p^{\prime} \mid p \in \mathcal{P}\left(U_{(2)}\right)\right\}
$$

if and only if $p$ is an edge of $K_{4,4}$. Since $\mathcal{P}\left(U_{(2)}\right)$ is coatomistic with infimum the intersection (Lemmas 4.4 and 4.1 ), the dual lattice $\mathcal{P}\left(U_{(2)}\right) *$ is atomistic with supremum the union. Thus $p \subset X$ lies in $\mathcal{P}\left(U_{(2)}\right)^{*}$ if and only if $p$ is a union (possibly empty) of edges of $K_{4,4}$.

It is not clear that any $p \subset X$ with $|p| \geqslant 5$ lies in $\mathcal{P}\left(U_{(2)}\right)^{*}$ because $p \cap V_{+1} \neq \varnothing$ and $p \cap V_{-1} \neq \varnothing$. Similarly, 68 of the 70 subsets of $X$ of cardinality four lie in $\mathcal{P}\left(U_{(2)}\right)^{*}$, the exceptions are $V_{+1}$ and $V_{-1}$. Dually, $\mathcal{P}\left(U_{(2)}\right)$ contains all $p \subset X$ with $|p| \leqslant 3$, which is a special case of Theorem 14 of $[34$.

We discuss ground spaces of frustration-free Hamiltonians [10, 41, 33, 16, 21], omitting detailed proofs. A $k$-local Hamiltonian $a \in U_{(k)}$ is frustration-free if $a$ has a sum representation (8.1) such that every ground state of $a$ is a ground state of $a(\nu) \otimes \mathbb{1}_{\nu^{\prime}},|\nu|=k$. The set $U_{(k)}^{\mathrm{ff}}$ of frustration-free $k$-local Hamiltonians is not a vector space. But the set of its ground projections, combined with the zero matrix, is a complete lattice, denoted $\mathcal{Q}\left(U_{(k)}\right)$, whose infimum is the intersection of images (3.1). The lattice is coatomistic, as the ground space of $a \in U_{(k)}^{\mathrm{ff}}$ is the intersection of ground spaces of the local terms $a(\nu) \otimes \mathbb{1}_{\nu^{\prime}},|\nu|=k$.

The dual lattice $\mathcal{Q}\left(U_{(k)}\right)^{*}$ is atomistic. In the commutative case $(3.3)$ the supremum of $\mathcal{Q}\left(U_{(k)}\right)^{*}$ is the union. For three bits, it is easy to see that the atoms of $\mathcal{Q}\left(U_{(2)}\right) *$ are the non-horizontal edges of $K_{4,4}$ in the vertex arrangement 


\begin{tabular}{cc}
$V_{+1}$ & $V_{-1}$ \\
\hline$(000)$ & $(111)$ \\
$(011)$ & $(100)$ \\
$(101)$ & $(010)$ \\
$(110)$ & $(001)$
\end{tabular}

The lattice $\mathcal{Q}\left(U_{(2)}\right)^{*}$ contains any $p \subset X$ with $|p| \geqslant 6$, because $p \cap V_{+1}$ and $p \cap V_{-1}$ contain at least two points each. The lattice $\mathcal{Q}\left(U_{(2)}\right)^{*}$ contains 48 of the 56 subsets $p \subset X$ with $|p|=5$, the eight subsets containing $V_{+1}$ or $V_{-1}$ are missing. Dually, $\mathcal{Q}\left(U_{(2)}\right)$ contains all $p \subset X$ with $|p| \leqslant 2$. The last observation follows also from Lemma 2 of [59], since there is a one-to-one correspondence between ground spaces of $U_{(k)}^{\mathrm{ff}}$ and support sets of probability distributions which, in the sense of [25], factor according to the log-linear model with generators $\{\nu \subset[N]:|\nu|=k\}$.

\section{Conclusion}

We proved two theorems concerning the lattice $\mathcal{P}(U)$ of ground projections of a vector space $U$ of hermitian matrices. First, Theorem 5.4 offers an equivalent form of the decision problem of whether a projection $p \in \mathcal{A}$ lies in $\mathcal{P}(U)$, see Section 1 . Second, Theorem 6.1 proves useful, in Section 9, to identify coatoms of $\mathcal{P}(U)$ for 3-bit Hamiltonians. Are the theorems useful in the non-commutative domain?

We are unable to decisively answer the question, but two remarks are in place. First, there does not exist any matrix in the space $U_{(2)}$ of 2-local 3-qubit Hamiltonians whose ground space has dimension seven. This is provable by showing the infeasibility of the real polynomial system from Remark 4.5 for $k=7$ (algebraic degree two). The FindInstance command of Wolfram Mathematica solves the problem in less than half a day on a $1.3 \mathrm{GHz}$ Intel Core i5 processor, using the cylindrical decomposition algorithm [9]. Second, an interesting problem to study is whether for every projection $p \in M_{8}$ of $\operatorname{rank} \operatorname{rk}(p) \leqslant 5$ either $K(p)=\{0\}$ or $\operatorname{dim} K(p)>1$ holds. If true, this would imply, with the help of the Theorems 5.4 and 6.1 , that all coatoms of $\mathcal{P}\left(U_{(2)}\right)$ have rank six, just as in the commutative case of three bits.

Acknowledgements. This article was put together 2016-2018 while the author was a postdoctoral scholar at IMECC, Unicamp, Brazil, and at QuIC, Université libre de Bruxelles, Belgium. He is glad of having been selected for a grant to join the trimester Analysis in Quantum Information Theory at Institut Henri Poincaré, Paris, France, in 2017. Among others, he thanks Alihuén García Pavioni, Arleta Szkoła, Aurelian Gheondea, Chi-Kwong Li, Eduardo Garibaldi, Frederic Shultz, Ilya Spitkovsky, Ivan Todorov, Jérémie Roland, Marcelo Terra Cunha, Michael Walter, Michał Horodecki, Paweł Horodecki, Ramis Movassagh, and Toby Cubitt for discussions concerning the article, lattices of projections, the quantum marginal problem, and ground spaces.

Stephan Weis

e-mail: maths@weis-stephan.de

Centre for Quantum Information and Communication

Ecole Polytechnique de Bruxelles

Université libre de Bruxelles

50 av. F.D. Roosevelt

1050 Bruxelles

Belgium 


\section{REFERENCES}

[1] M. Aigner, Combinatorial Theory, Berlin, Heidelberg: Springer-Verlag, 1997.

[2] E. M. Alfsen, F. W. Shultz, State Spaces of Operator Algebras: Basic Theory, Orientations, and $C^{*}$-Products, Boston: Birkhäuser, 2001.

[3] M. Altunbulak, A. Klyachko, The Pauli Principle Revisited, Communications in Mathematical Physics, 282 (2008), 287-322.

[4] S.-I. Amari, Information geometry on hierarchy of probability distributions, IEEE Transactions on Information Theory 47 (2001), 1701-1711.

[5] G. Averkov, V. Kaibel, S. Weltge, Maximum semidefinite and linear extension complexity of families of polytopes, Mathematical Programming 167 (2018), 381-394.

[6] N. Ay, E. Olbrich, N. Bertschinger, J. Jost, A geometric approach to complexity, Chaos 21 (2011), 037103.

[7] N. Ay, A. Knauf, Maximizing multi-information, Kybernetika 42 (2006), 517-538.

[8] G. P. Barker, Faces and duality in convex cones, Linear and Multilinear Algebra 6 (1978), 161-169.

[9] S. Basu, R. Pollack, M.-F. Roy, Algorithms in Real Algebraic Geometry, 2nd ed., Berlin: Springer, 2006.

[10] N. de Beaudrap, T.J. Osborne, J. Eisert, Ground states of unfrustrated spin Hamiltonians satisfy an area law, New Journal of Physics 12 (2010), 095007.

[11] I. Bengtsson, K. Życzkowski, Geometry of Quantum States, 2nd ed., Cambridge: Cambridge University Press, 2017.

[12] I. Bengtsson, S. Weis, K. Życzkowski, Geometry of the set of mixed quantum states: An apophatic approach, in: Geometric Methods in Physics, P. Kielanowski et al. (eds.), Basel: Birkhäuser, 2013, 175-197.

[13] G. Birkhoff, Lattice Theory, 3rd ed., Providence, R.I.: AMS, 1967.

[14] A. Böttcher, I. M. Spitkovsky, A gentle guide to the basics of two projections theory, Linear Algebra and its Applications 432 (2010), 1412-1459.

[15] O. Bratteli, D. W. Robinson, Operator Algebras and Quantum Statistical Mechanics 1, 2nd ed., Berlin, Heidelberg: Springer-Verlag, 1987.

[16] J. Chen, Z. Ji, D. Kribs, Z. Wei, B. Zeng, Ground-state spaces of frustration-free Hamiltonians, Journal of Mathematical Physics 53 (2012), 102201.

[17] J. Chen, Z. Ji, M. B. Ruskai, B. Zeng, D.-L. Zhou, Comment on some results of Erdahl and the convex structure of reduced density matrices, Journal of Mathematical Physics 53 (2012), 072203.

[18] M. Christandl, B. Doran, S. Kousidis, M. Walter, Eigenvalue distributions of reduced density matrices, Communications in Mathematical Physics 332 (2014), 1-52.

[19] A. Coleman, Structure of Fermion density matrices, Reviews of Modern Physics 35 (1963), 668-686.

[20] T. Cubitt, A. Montanaro, Complexity classification of local Hamiltonian problems, SIAM Journal on Computing 45 (2016), 268-316.

[21] A. S. Darmawan, S.D. Bartlett, Graph states as ground states of two-body frustration-free Hamiltonians, New Journal of Physics 16 (2014), 073013.

[22] M. Develin, S. Sullivant, Markov bases of binary graph models, Annals of Combinatorics 7 (2003), 441-466.

[23] R. M. Erdahl, The convex structure of the set of $N$-representable reduced 2-matrices, Journal of Mathematical Physics 13 (1972), 1608-1621.

[24] S. Fiorini, S. Massar, S. Pokutta, H. R. Tiwary, R. de Wolf, Linear vs. semidefinite extended formulations: Exponential separation and strong lower bounds, in: STOC '12, New York: ACM, 2012, 95-106.

[25] D. Geiger, C. Meek, B. Sturmfels, On the toric algebra of graphical models, The Annals of Statistics 34 (2006), 1463-1492.

[26] A. Gheondea, S. Gudder, P. Jonas, On the infimum of quantum effects, Journal of Mathematical Physics 46 (2005), 062102.

[27] D. J. Griffiths, Introduction to Quantum Mechanics, 2nd ed., Upper Saddle River, NJ: Pearson Prentice Hall, 2005. 
[28] B. Groisman, S. Popescu, A. Winter, Quantum, classical, and total amount of correlations in a quantum state, Physical Review A 72 (2005), 032317.

[29] D. Gross, M. Müller, R. Colbeck, O.C. O. Dahlsten, All reversible dynamics in maximally nonlocal theories are trivial, Physical Review Letters 104 (2010), 080402.

[30] O. Gühne, M. Kleinmann, T. Moroder, Analysing Multiparticle Quantum States, in: Quantum [Un]Speakables II, R. Bertlmann, A. Zeilinger (eds.), Cham: Springer, 2017, 345-364.

[31] A.S. Holevo, Quantum Systems, Channels, Information: A Mathematical Introduction, Berlin: De Gruyter, 2012.

[32] R. A. Horn and C. R. Johnson, Matrix Analysis, 2nd ed., Cambridge: Cambridge University Press, 2012.

[33] Z. Ji, Z. Wei, B. Zeng, Complete characterization of the ground-space structure of two-body frustration-free Hamiltonians for qubits, Physical Review A 84 (2011), 042338.

[34] T. Kahle, Neighborliness of marginal polytopes, Contributions to Algebra and Geometry 51 (2010), 45-56.

[35] J. Kempe, A. Kitaev, O. Regev, The complexity of the local Hamiltonian problem, SIAM Journal on Computing 35 (2006), 1070-1097.

[36] A. Kitaev, A. Shen, M. Vyalyi, Classical and Quantum Computation, Graduate Studies in Mathematics 47, Providence: AMS, 2002.

[37] F. Kubo, T. Ando, Means of positive linear operators, Mathematische Annalen 246 (1980), 205-224.

[38] N. Linden, S. Popescu, W. Wootters, Almost every pure state of three qubits is completely determined by its two-particle reduced density matrices, Physical Review Letters 89 (2002), 207901.

[39] Y.-K. Liu, Consistency of local density matrices is QMA-complete, Lecture Notes in Computer Science 4110, Berlin, Heidelberg: Springer, 2006, 438-449.

[40] R. Loewy, B.-S. Tam, Complementation in the face lattice of a proper cone, Linear Algebra Appl 79 (1986), 195-207.

[41] R. Movassagh, E. Farhi, J. Goldstone, D. Nagaj, T. J. Osborne, P. W. Shor, Unfrustrated qudit chains and their ground states, Physical Review A 82 (2010), 012318.

[42] V. Müller, The joint essential numerical range, compact perturbations, and the Olsen problem, Studia Mathematica 197 (2010), 275-290.

[43] Y. Nesterov, A. Nemirovskij, Interior-Point Polynomial Algorithms in Convex Programming, 3. printing, Philadelphia: SIAM, 2001.

[44] S. Niekamp, T. Galla, M. Kleinmann, O. Gühne, Computing complexity measures for quantum states based on exponential families, Journal of Physics A: Mathematical and Theoretical 46 (2013), 125301.

[45] S. A. Ocko, X. Chen, B. Zeng, B. Yoshida, Z. Ji, M. B. Ruskai, I. L. Chuang, Quantum codes give counterexamples to the unique preimage conjecture of the $N$-representability problem, Phys Rev Lett 106 (2011), 110501.

[46] V. I. Paulsen, Completely Bounded Maps and Operator Algebras, Cambridge: Cambridge University Press, 2002.

[47] M. Ramana, A.J. Goldman, Some geometric results in semidefinite programming, J Global Optim 7 (1995), 33-50.

[48] J. Rauh, Finding the maximizers of the information divergence from an exponential family, IEEE Transactions on Information Theory 57, 3236-3247 (2011).

[49] R. T. Rockafellar, Convex Analysis, Princeton: Princeton University Press, 1970.

[50] L. Rodman, I. M. Spitkovsky, A. Szkoła, S. Weis, Continuity of the maximum-entropy inference: Convex geometry and numerical ranges approach, Journal of Mathematical Physics $\mathbf{5 7}$ (2016), 015204.

[51] J. J. Sakurai, J. Napolitano, Modern Quantum Mechanics, 2nd ed., Boston: Addison-Wesley, 2011.

[52] A. Sawicki, M. Walter, M. Kuś, When is a pure state of three qubits determined by its singleparticle reduced density matrices?, Journal of Physics A: Mathematical and Theoretical 46 (2013), 055304.

[53] R. Schneider, Convex Bodies: The Brunn-Minkowski Theory, 2nd ed., New York: Cambridge University Press, 2014.

[54] S. Weis, Quantum convex support, Linear Algebra Appl 435 (2011), 3168-3188. 
[55] S. Weis, A note on touching cones and faces, Journal of Convex Analysis 19 (2012), 323-353.

[56] S. Weis, Continuity of the maximum-entropy inference, Communications in Mathematical Physics 330 (2014), 1263-1292.

[57] S. Weis, Operator systems and convex sets with many normal cones, Journal of Convex Analysis 25 (2018), 41-64.

[58] S. Weis, A. Knauf, Entropy distance: New quantum phenomena, Journal of Mathematical Physics 53 (2012), 102206.

[59] S. Weis, A. Knauf, N. Ay, M.-J. Zhao, Maximizing the divergence from a hierarchical model of quantum states, Open Systems \& Information Dynamics 22 (2015), 1550006.

[60] B. Zeng, X. Chen, D.-L. Zhou, X.-G. Wen, Quantum Information Meets Quantum MatterFrom Quantum Entanglement to Topological Phase in Many-Body Systems, arXiv:1508.02595 [cond-mat.str-el]

[61] D. Zhou, Irreducible multiparty correlations in quantum states without maximal rank, Physical Review Letters 101 (2008), 180505.

[62] G. M. Ziegler, Lectures on Polytopes, New York: Springer-Verlag, 1995. 